
UM SISTEMA EM UM CAMPO EXTERNO

\title{
SOME REMARKS ON THE CHEMICAL POTENTIAL OF A SYSTEM IN AN EXTERNAL FIELD
}

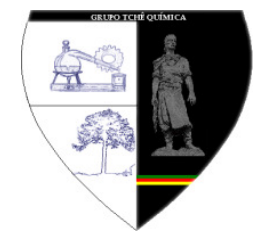

\author{
SALVESTRINI, Stefano ${ }^{1,2 *}$; AMBROSONE, Luigi ${ }^{3}$; IOVINO, Pasquale ${ }^{1,2} ;$ CAPASSO, Sante ${ }^{2}$ \\ ${ }^{1}$ Department of Environmental, Biological and Pharmaceutical Sciences and Technologies, Università degli \\ Studi della Campania "Luigi Vanvitelli", via Vivaldi 43, 81100 Caserta, Italy \\ ${ }^{2}$ Environmental Technologies, University Spin-Off of University of Campania "Luigi Vanvitelli”, Via Vivaldi, 43, \\ Caserta, Italy \\ ${ }^{3}$ Department of Medicine and Health Sciences "V. Tiberio", University of molise 86100 Campobasso, Italy \\ * Corresponding author \\ e-mail: stefano.salvestrini@unicampania.it
}

Received 21 September 2018; received in revised form 22 October 2018; accepted 22 October 2018

\section{RESUMO}

A mudança do potencial químico fornece um critério para prever a espontaneidade de qualquer processo físico e químico. Se for pedido para calcular a mudança do potencial químico de um sistema no qual várias forças variam, um estudante pode achar a tarefa bastante complicada à primeira vista. No entanto, o potencial químico é uma função do estado. Esta propriedade permite uma definição precisa da contribuição de cada força para o potencial químico quando todos os outros parâmetros relevantes são mantidos constantes. A alteração total do potencial químico pode ser facilmente calculada somando as contribuições acima. Após uma breve revisão do papel desempenhado por alguns parâmetros do sistema, como atividade $(a)$ dos componentes, temperatura $(T)$, pressão $(p)$ e tensão superficial $(\gamma)$, assim como campos externos como, por exemplo, gravitacional $(M g h)$, centrifugo $(M c \rho)$ e campo elétrico $\left(F z_{i_{-}}\right)$, uma equação para a computação do potencial químico $(\mu)$ incluindo todas as contribuições acima é relatado: $\mu_{i}=\mu_{i}{ }^{\prime}+R T \ln a_{i}+\bar{V}_{i}(p-p \quad)-$ $\bar{S}_{i}(T-T)+M g h+M c \rho+F z_{i},+2 \gamma \bar{V}_{i} \frac{1}{r}$, onde $\mu_{i}^{\prime \prime}$ se refere não soffpente para $\mathrm{p}=\mathrm{p}^{\circ}=1$ bar, mas também para um valor escolhido de $T, h, \hat{h}, \Phi$ e $r$. Finalmente, exemplos aplicalj.jos são ilustrados.

Palavras-chave: Potencial Quínff|co; Função de estado; Campo externp.

ABSTRACT<smiles>[AlH2]C=[AlH2]</smiles>

The chemical potential change provides a criterion for predicting the spontaneity of any physical and chemical process. If asked to calculate the chemical potential change of a system in which several forces vary, a student might find the task quite complicate at first glance. However, the chemical potential is a state function. This property permits a precise definition of the contribution of each force to the chemical potential when all other relevant parameters are kept constant. The total chemical potential change can easily be calculated by summing up the above contributions. After a brief review of the role played by some parameters of the system, like activity $(a)$ of the components, temperature $(T)$, pressure $(p)$ and surface tension $(\gamma)$, as well as of external fields, i.e. gravitational $(M g h)$, centrifugal $(M c \rho)$ and electric field $\left(F z_{i}\right)$, an equation for the computation of the chemical potential $(\mu)$ including all the above contributes is reported: $\mu_{i}=\mu_{i}{ }^{\prime}+R T \ln a_{i}+\bar{V}_{i}(p-p \quad)-$ $\bar{S}_{i}(T-T)+M g h+M c \rho+F z_{i},+2 \gamma \bar{V}_{i} \frac{1}{r}$, where $\mu_{i}^{\circ}$ refers not only t $\mathrm{f} \| \mathrm{p}=\mathrm{p}^{\circ}=1$ bar but also to a chosen value of $T, h, \rho, \Phi$ and $r$. Finally, applicative examples are illustrated.

Keywords: Chemical potential; $\$$ fate function; External field.

PERIÓDICO TCHÊ QUÍMICA • www.periodico.tchequimica.com • Vol. 16 N. 31.

- ISSN 1806-0374 (impresso) • ISSN 1806-9827 (CD-ROM) • ISSN 2179-0302 (meio eletrônico)

(c) 2019. Porto Alegre, RS. Brasil 


\section{INTRODUCTION}

As reported in thermodynamics textbooks (Atkins et al. 2017; Capasso 2012; De Voe, 2014), Gibbs equations for open systems have the following forms:

$$
\begin{aligned}
& d U=T d S-p d V+\sum_{i=1}^{k} \mu_{i} d n_{i} \\
& d H=T d S+V d p+\sum_{i=1}^{k} \mu_{i} d n_{i} \\
& d A=-S d T-p d V+\sum_{i=1}^{k} \mu_{i} d n_{i} \\
& d G=-S d T+V d p+\sum_{i=1}^{k} \mu_{i} d n_{i}
\end{aligned}
$$

where $\mathrm{U}, \mathrm{T}, \mathrm{S}, \mathrm{p}, \mathrm{V}, \mathrm{H}, \mathrm{A}$ and $\mathrm{G}$ are the internal energy, thermodynamic temperature, entropy, pressure, volume, enthalpy, Helmholtz energy and Gibbs energy, respectively, and $\mu_{i}$ the chemical potential (Gibbs, 1878) of the system components. The "potential" is ability of a system to perform work. $\sum_{i=1}^{k} \mu_{i} d n_{i}$ is frequently referred to as the "chemical work". It follows from eq.(4) that the chemical potential of a component in a solution is defined by the relation

$\mu_{i}=\left(\frac{\partial G}{\partial n_{i}}\right)_{T, p, n_{j \neq} n_{i}}=\bar{G}_{i}$

where $\bar{G}_{i}$ is the partial molar Gibbs energy and $\mu_{i}$ the rate at which the (extensive) Gibbs energy of the solution increases as the number of moles (extensive) of the component in question increases; $\mu_{i}$ is expressed in energy units per unit mass (Joule/kg), per mole (Joule/mole), or per molecule of the substance considered: in this paper we will refer to moles. Other relations that define the chemical potential can be obtained from eqs.(1) to (3) as follows:

$$
\begin{aligned}
& \mu_{i}=\left(\frac{\partial U}{\partial n_{i}}\right)_{S, V, n_{j \neq} n_{i}} ; \mu_{i}=\left(\frac{\partial H}{\partial n_{i}}\right)_{S, p, n_{j \neq} n_{i}} ; \mu_{i}= \\
& \left(\frac{\partial A}{\partial n_{i}}\right)_{T, V, n_{j \neq} n_{i}} \text { (Eq.6) }
\end{aligned}
$$

One of the most frequent uses of the chemical potential is to define the equilibrium or distribution criterion of a component between two or more phases. It measures the "escaping tendency" for a component in a solution, i.e. the tendency of a compound to escape by moving to another point of the same phase or another phase, or by reacting.

Before being introduced to the notion of chemical potential, undergraduate students usually have already met different types of potential, notably the electric potential and gravitational potential. In each case the product of the potential difference between two points and the amount of what is transferred (electric charges and matter for the electric and gravitational potential, respectively) gives the work (or energy) necessary for the shift to take place in a reversible manner, or produced by the shift. Likewise, if between two points or two states $\alpha$ and $\beta$ of a system there is a difference in the chemical potential of the compound $\mathrm{i}\left(\mu_{i}^{\beta}\right.$ and $\left.\mu_{i}^{\alpha}\right)$, the product between $\left(\mu_{i}^{\beta}-\mu_{i}^{\alpha}\right)$ and $n_{i}$ is the minimum work necessary for the transition, by a chemical or physical process, of $n$ moles of the compound i from $\alpha$ to $\beta$ (or the maximum amount of non-expansion work that can be extracted). This relation between chemical potential and work allows the assessment of the contribute of external fields on $\mu$ (Yeats et al., 2017; Job and Herrmann, 2006; Guangze and Janjia, 2018; Lepori et al., 2018; Kaptay, 2017; Sevilla and Olivarez-Quinoz, 2012; Salvestrini et al., 2014; Salvestrini et al., 2015).

From chemical thermodynamics we know that the chemical potential is related to the activity of a compound in a condensed phase (or to the concentration for an ideal solution, or to the fugacity, or pressure, for gas) by the relation:

$\mu_{i}=\mu_{i}^{\circ}+R T \ln a_{i}$

where $\mu_{i}^{\circ}$ is the chemical potential of $i$ in the standard state. This equation supposes that all the other variables that affect thermodynamic properties of $i$ are constant.

The aim of this paper is to put together the contributes of all the variables involved in a single equation.

\section{CHEMICAL POTENTIAL AS AFFECTED BY DIFFERENT PARAMETERS}

Gibbs energy is a function of all the variables that affect the system:

$G=f\left(X_{1}, X_{2}, \ldots \ldots, X_{n}\right)$

From (Eq.5) and (Eq.8) we obtain, for the compound $i$ in a system characterised by $n$ independent variables: 
$d \mu_{i}=\sum_{1}^{n}\left(\frac{d \bar{G}_{i}}{d X_{m}}\right) d X_{m}$

where $X_{m}$ is the $m^{\text {th }}$ variable. Let us now consider a system in which $\mu_{i}$ is affected by the activity (or fugacity for a gaseous system) $\left(a_{i}\right)$, pressure $(p)$, and temperature $(T)$, whereas all the other variables are constant; from eq.(9) we obtain:

$d \mu_{i}=\left(\frac{d \bar{G}_{i}}{d a_{i}}\right)_{p, T, n_{j \neq} n_{i}} d a_{i}+\left(\frac{d \bar{G}_{i}}{d p}\right)_{a_{i}, T, n_{j \neq} n_{i}} d p+$

$\left(\frac{d \bar{G}_{i}}{d T}\right)_{a_{i}, p, n_{j \neq} n_{i}} d T$

$d \mu_{i}=\frac{R T}{a_{i}} d a_{i}+\bar{V}_{i} d p-\bar{S}_{i} d T$

where $\bar{S}_{i}$ and $\bar{V}_{i}$ are the molar entropy and molar volume, respectively.

The change in the chemical potential between state 1 and state 2 due to activity, temperature and pressure can therefore be expressed as:

$\mu_{i}(2)-\mu_{i}(1)=\int_{a_{i, 1}}^{a_{i, 2}} \frac{R T}{a_{i}} d a_{i}+\int_{p_{1}}^{p_{2}} \bar{V}_{i} d p-\int_{T_{1}}^{T_{2}} \bar{S}_{i} d T$

If other forces act on the system, e.g. a gravitational field or electromagnetic field (Ambrosone and Fontana, 2005) their contribution to the change in Gibbs energy and chemical potential should be added into eqs.(4, $10,11)$. Let us examine the contribution of gravitational field, centrifugal field, electric field and surface tension.

It is known that the differential chemical potential of an object of molar mass $M$ in a gravitation field with gravitational acceleration $g$ varies with the height $h$ (keeping constant all the other parameters that affect Gibbs energy) as follows (Han and Wang, 2013):

$d \mu_{i}=M g d h$

The same applies for a centrifugal field:

$d \mu_{i}=\operatorname{Mcd} \rho$

where $c$ is the centrifugal acceleration and $\rho$ the radial distance from the rotation axis.

The differential chemical potential for charged species can be expressed as:

$d \mu_{i}=F z_{i} d \Phi$ where $F$ is the Faraday constant, $z_{i}$ the charge of ion $\mathrm{i}$, and $\Phi$ the electric field potential.

It is known that in a closed container saturated with the vapour of a specific liquid, the smaller drops disappear while the larger ones increase in size. This is due to the fact that Gibbs energy varies with the surface area (once again by keeping constant all the other parameters) according to the relation:

$d G=\gamma d s$

where $y$ is the surface tension and $s$ the surface area. For a spherical drop, we may express the rate of change of $s$ with volume $\mathrm{V}$ as

$\frac{d s}{d V}=\frac{8 \pi r d r}{4 \pi r^{2} d r}=\frac{2}{r}$

which leads to

$d s=\frac{2}{r} d V=\frac{2}{r} \bar{V}_{i} d n_{i}$

where $r$ is radius of the drop. It follows that an infinitesimal change in the chemical potential arising from an infinitesimal change in the surface area is (Lewis and Randall, 1961)

$d \mu_{i}=2 \gamma \bar{V}_{i} d \frac{1}{r}$

Therefore, the total chemical potential change due to changes in the activity, pressure, temperature, gravitational field, centrifugal field, electric field and surface tension is given by

$d \mu_{i}=\left(\frac{d \bar{G}_{i}}{d a_{i}}\right)_{p, T, h, \rho, \Phi, \mathbf{s}, n_{j \neq} n_{i}} d a_{i}+$

$\left(\frac{d \bar{G}_{i}}{d p}\right)_{a_{i}, T, h, \rho, \Phi, \mathrm{s}, n_{j \neq} n_{i}} d p+\left(\frac{d \bar{G}_{i}}{d T}\right)_{a_{i, p}, h, \rho, \Phi, \mathrm{s}, n_{j \neq} n_{i}} d T+$

$\left(\frac{d \bar{G}_{i}}{d h}\right)_{a_{i}, p, T, \rho, \Phi, \mathrm{s}, n_{j \neq} n_{i}} d h+\left(\frac{d \bar{G}_{i}}{d \rho}\right)_{a_{i}, p, T, h, \Phi, \mathrm{s}, n_{j \neq} n_{i}} d \rho+$

$\left(\frac{d \bar{G}_{i}}{d \Phi}\right)_{a_{i}, p, T, h, \rho, \mathrm{s}, n_{j \neq} n_{i}} d \Phi+\left(\frac{d \bar{G}_{i}}{d s}\right)_{a_{i}, p, T, h, \rho, \Phi, n_{j \neq} n_{i}} d \mathrm{~s}$

(Eq.20)

$\int_{\mu_{i}(1)}^{\mu_{i}(2)} d \mu_{i}=\mu_{i}(2)-\mu_{i}(1)=\int_{a_{i, 1}}^{a_{i, 2}} \frac{R T}{a_{i}} d a_{i}+$

$\int_{p_{1}}^{p_{2}} \bar{V}_{i} d p-\int_{T_{1}}^{T_{2}} \bar{S}_{i} d T+\int_{h_{1}}^{h_{2}} M g d h+\int_{\rho_{1}}^{\rho_{2}} M c d \rho+$

$\int_{\Phi_{1}}^{\Phi_{2}} F z_{i} d \Phi+\int_{\frac{1}{r_{1}}}^{\frac{1}{r_{2}}} 2 \gamma \bar{V}_{i} d \frac{1}{r}$

If we assume that $\mu_{i}^{\circ}$ refers not only to $p=p^{\circ}=1$ bar but also to a chosen standard state of $a\left(a^{\circ}=\right.$ 1), $T\left(\right.$ e.g. $\left.T^{\circ}=25^{\circ} \mathrm{C}\right), h\left(h^{\circ}=0\right), \rho\left(\rho^{\circ}=0\right), \Phi\left(\Phi^{\circ}\right.$ $=0)$ and $r\left(r^{\circ}=+^{\infty}\right.$, i.e. plane surface $)$, and 
indicating this state with the symbol $\mu_{i}{ }^{\circ}$, we have from eq.(16) that the chemical potential of $i$ at some $a_{i}, p, T, h, \rho, \Phi$ and $r$ is:

$\int_{\mu_{i}}^{\mu_{i}}, d \mu_{i}=\int_{a_{i}}^{a_{i}} \frac{R T}{a_{i}} d a_{i}+\int_{p}^{p} \bar{V}_{i} d p-\int_{T}^{T} \bar{S}_{i} d T+$ $\int_{h}^{h} M g d h+\int_{\rho}^{\rho} M c d \rho+\int \downarrow F z_{i} d$ $++\int_{\frac{1}{r}}^{\frac{1}{r}} 2 \gamma \bar{V}_{i} d \frac{1}{r}$ $\left.\mu_{i} \mid=\mu_{i}{ }^{\prime}+R T \ln a_{i}+\bar{V}_{i}(p) p \quad\right)-\$(T-T)+$ $M g h+M c \rho+F z_{i}+2 \gamma \bar{V}_{i} \frac{1}{r} \quad$ (Eq.23)

where $\bar{V}_{i}, \bar{S}_{i}, \mathrm{~g}$, and $\mathrm{\gamma}$ have been assumed constant relative to $\mathrm{p}, \mathrm{T}, \mathrm{h}, \mathrm{g}, \rho$ and $\mathrm{r}$, respectively.

The above equation allows an easy computation of changes of $\mu$ when different parameters such as electric field, gravity, temperature and pressure act on the system.

Let us examine possible applications of the equation above.

\section{EXAMPLES}

Example 1. Consider an anionic polymer, molecular mass $=100,000 \mathrm{~g} \mathrm{~mol}^{-1}$, in the sodium form $\left(P^{n-} N a_{n}^{+}\right)$. Its aqueous solution, $100 \mathrm{mg} \mathrm{L}^{-1}$ is put in an osmometer, with a membrane permeable to $\mathrm{Na}^{+}$ion and water molecules but not to $\mathrm{P}^{\mathrm{n}-}$ anions. A constant pressure of 10.0 bar is applied to the polymer solution (phase $\alpha$ ), the pressure on the other side (phase $\beta$ ) being 1.0 bar. At the equilibrium, $\mathrm{T}=25^{\circ} \mathrm{C}$, the membrane potential (electrical potential difference between phase $\beta$ and phase $\alpha$ is $0.100 \mathrm{~V}$ and the sodium concentration in the phase $\alpha$ is $0.01 \mathrm{M}$. Knowing that the partial molar volume of $\mathrm{Na}^{+}\left(\bar{V}_{N a^{+}}\right)=-$ $6.62 \mathrm{~mL} \mathrm{~mol}^{-1}$ (Marcus, 2009), calculate the concentration of $\mathrm{Na}^{+}$in the phase $\beta$ at the equilibrium.

Solution: At the equilibrium the chemical potential of $\mathrm{Na}^{+}$is, of course, the same on either side of the osmometer, i.e. $\mu_{\alpha, N a^{+}}=\mu_{\beta, N a^{+}}$. Under constant electric, gravitational and centrifugal fields (or in their absence), under constant temperature, and assuming that the solutions on both sides of the membrane have planar surface and ideal behaviour, eq.(23) gives:

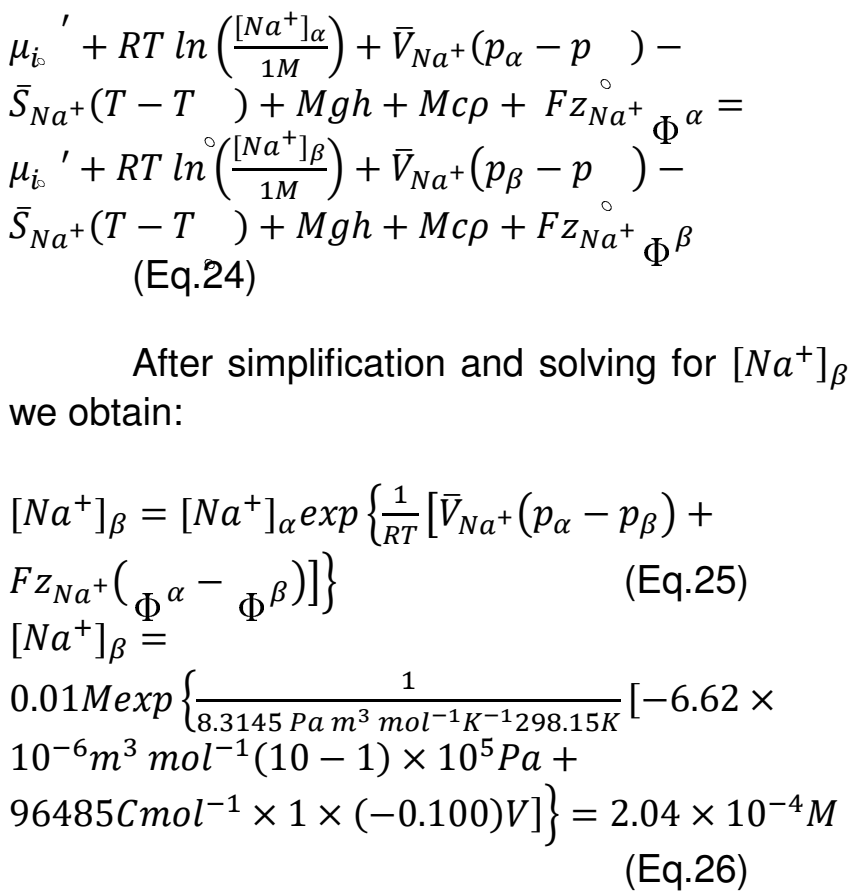

Example 2. In a chemical plant, two tanks contain water kept at constant temperature and pressure: the first tank at $\mathrm{T}=30{ }^{\circ} \mathrm{C}$ and the second at $\mathrm{T}=50^{\circ} \mathrm{C}$; the first is under a pressure of $1.0 \mathrm{bar}$, the latter of $10 \mathrm{bar}$. If the two tanks are separated by a permeable membrane to water, predict the direction of flow.

Standard molar entropy of water (at 298K) $\left(\bar{S}^{\circ}\right)$ : = $69.91 \mathrm{~J} \mathrm{~K}^{-1} \mathrm{~mol}^{-1}$

Molar volume of water $(\bar{V}): 18 \mathrm{~mL} \mathrm{~mol}^{-1}$

Molar heat capacity of water $\left(\bar{C}_{p}\right): 75.2 \mathrm{~J} \mathrm{~K}^{-1} \mathrm{~mol}^{-}$ 1.

Solution: In this example, $\mu$ depends only on pressure and temperature, hence from eq.(22) we obtain:

$\int_{\mu_{i}}^{\mu_{i}}, d \mu_{i}=\int_{p}^{p} \bar{V} d p-\int_{T}^{T} \bar{S} d T$

The effect of temperature on entropy can be taken into account, assuming that $\bar{C}_{p}$ does not vary with temperature, by:

$\bar{S}=\bar{S}^{\circ}+\bar{C}_{p} \ln \frac{T}{T^{\circ}}$

By substituting eq.(28) into eq.(27) and integrating, we get

$$
\begin{aligned}
& \mu_{1}=\mu_{w}{ }^{\prime}+\bar{V}\left(p_{1}-p\right)-\bar{S} \quad\left(T_{1}-T\right)- \\
& \bar{C}_{p} T_{1}\left(\ln \frac{T_{1}}{T}-1\right)
\end{aligned}
$$

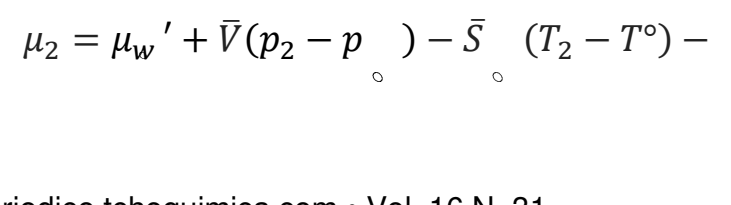


$\bar{C}_{p} T_{2}\left(\ln \frac{T_{2}}{T^{\circ}}-1\right)$

where $\mu_{1}$ and $\mu_{2}$ are the chemical potential of water in the first and second tank, respectively, and $\mu_{w}{ }^{\prime}$ is the standard chemical potential of water.

The difference in chemical potential between the two tanks is

$\mu_{2}-\mu_{1}=-1456 \mathrm{~J} \mathrm{~mol}^{-1}<0$

Therefore, although the pressure in the second tank is much higher than that in the first tank, water will flow in the opposite direction, i.e. from the first (cold) towards the second (hot) tank. In this example, the decrease in the chemical potential with temperature overcomes its increase with pressure. A similar direction of mass trasport (from cold to hot area) may be observed, for example, for the thermodiffusion of molecules with sufficient high molar entropy (Kocherginsky and Gruebele, 2016; Barragàn and Kjelstrup, 2017).

\section{CONCLUSIONS:}

We have shown that the calculation of chemical potential as simultaneously affected by several thermodynamic parameters, can be easily calculated by summing up the individual contribution of each parameter.

\section{REFERENCES:}

1. Ambrosone L, Fontana F. Eur Phys J B 2005, 48, 537-545.

2. Atkins P, de Paula J, Keeler J. In: Atkins' Physical Chemistry. OUP Oxford eds. (2017) ISBN: 9780198769866

3. Barragán VM, Kjelstrup S. Thermoosmosis in Membrane Systems: A Review.(2017) Journal of Non-Equilibrium Thermodynamics, 42(3), pp. 217-236

4. Capasso S. In: La Chimica Fisica Attraverso Esercizi. Loghia eds, Italy, 2012 ISBN: 9788895122205.

5. Cavalcanti E, Castro E, Linhares CA, Malbouisson APC, Influence of external magnetic field, finite-size effects and chemical potential on the phase transition of a complex scalar field, Eur. Phys. J. C
(2017) 77: 711

6. DeVoe H. In: Thermodynamics and Chemistry, copyright 2014 by Howard DeVoe (2017) http://www2.chem.umd.edu/thermobook.

7. Gibbs JW. Am J Sci 1878, 16, 441-458.

8. Guangze $\mathrm{H}$ and Jianjia $\mathrm{M}$, Extension of Gibbs-Duhem equation including influences of external fields, Continuum and Thermodynamics, 30 (2018), 817-823

9. Han $\mathrm{G}$, Wang $\mathrm{H}$, Generalized expression of chemical potential with influence of external fields and its applications: Effect of charged particles on droplet condensation, Fluid Phase Equilibria, 338, (2013), 269-273

10. Job $G$ and Herrmann F, Chemical potential a quantity in search of recognition Eur. J Phys 27 (2) 2006

11. Kaptay G, A new paradigm on the chemical potentials of components in multi-component nano-phases within multi-phase systems, RSC Advances, 2017, 7, 41241-41253

12. Kocherginsky N, Gruebele M. Mechanical approach to chemical transport. PNAS (2016) 113 (40) 11116-11121.

13. Lepori L, Maraga A, Celi A, Dell'Anna L, Trombettoni A, Effective Control of Chemical Potentials by Rabi Coupling with RF-Fields in Ultracold Mixtures, Condens. Matter 2018, 3, 14; doi:10.3390/condmat3020014

14. Lewis GN, Randall M. In: Thermodynamics. New York, McGraw-Hill (1961) ISBN: 9780070376229.

15. Marcus Y. J Phys Chem B 2009, 113(30), 10285-10291.

16. Salvestrini S, Leone V, lovino P, Canzano $S$, Capasso S. Considerations about the correct evaluation of sorption thermodynamic parameters from equilibrium isotherms. Journal of Chemical Thermodynamics, 2014, 68: 310-316. 
17. Salvestrini $S$, Vanore $P$, lovino $P$, Leone V, Capasso S. Adsorption of Simazine and Boscalid onto acid-activated natural clinoptilolite. Environmental Engineering and Management Journal, 2015, 14(7): 1705-1712.

18. Sevilla FJ and Olivares-Quiroz L, Chemical potential for the interacting classical gas and the ideal quantum gas obeying a generalized exclusion principle, Eur. J. Phys. 33 (2012) 709-722.

19. Yeats $A L$, Mintun $P J$, Pan $Y$, Richardella A, Buckley BB, Samarth N, Awschalom DD, PNAS 26, 2017114 (39) 1037910383

PERIÓDICO TCHÊ QUÍMICA • www.periodico.tchequimica.com • Vol. 16 N. 31

- ISSN 1806-0374 (impresso) • ISSN 1806-9827 (CD-ROM) • ISSN 2179-0302 (meio eletrônico)

(C) 2019. Porto Alegre, RS. Brasil 